\title{
Platelet-Rich Plasma (PRP) in a Post Radiotherapy Sternal Ulcer in a Patient with Hodgkin's Lymphoma
}

\author{
Fioramonti $\mathbf{P}^{1}$, Fino $\mathbf{P}^{1^{\star}}$, Ferrazza $\mathbf{G}^{2}$, Capria $\mathbf{V}^{2}$ and Onesti $\mathbf{M G}^{1}$ \\ ${ }^{1}$ Department of Plastic, Reconstructive and Aesthetic Surgery, University of Rome "Sapienza", Policlinico Umberto I, Viale del Policlinico, 155, 00161, Rome, Italy \\ ${ }^{2}$ Department of Cellular Biotechnologies and Hematology, University of Rome "Sapienza”, Policlinico Umberto I, Viale del Policlinico, 155, 00161, Rome, Italy
}

"Corresponding author: Pasquale Fino, Department of Plastic, Reconstructive and Aesthetic Surgery, University of Rome " La Sapienza”, Policlinico Umberto I, Viale Pantelleria, 35, Scala B, Interno 1/A, 00141, Rome, Italy, Tel: 39-3334571756; Fax: +39-06/491525/+39-06 64491523; E-mail: pasquale.fino@gmail.com, pasquale.fino@uniroma1.it

Received date: Aug 21 2014, Accepted date: Aug 18, 2015, Publication date: Aug 21, 2015

Copyright: (c) 2015 Fioramonti P, et al. This is an open-access article distributed under the terms of the Creative Commons Attribution License, which permits unrestricted use, distribution, and reproduction in any medium, provided the original author and source are credited.

\begin{abstract}
Radiotherapy is an essential part of the multidlevel treatment for Hodgkin's Lymphoma. However but it is responsible for several adverse effects on different tissues. The tissue damage may occur immediately or over a prolonged period of time. A 33 years old woman, with a diagnosis of Hodgkin lymphoma, nodular sclerosis histotype, II grade according to $\mathrm{WHO}$ classification, stage $2 \mathrm{~A}$ has been examined for this study. At the physical examination, the ulcer was of $6 \times 5 \mathrm{~cm}, 2 \mathrm{~cm}$ deep, of circular shape, with uneven margins. There was rash around the lesion too. The wound was infected with abundant serous and fibrinous exudate in the center and squamous-crostous formations around the center itself. The lesion was bleeding and smelled bad. Platelet gel has been applied once a week with a control after 3 days to check and evaluate the conditions of the medication.

The situation improved after the first two applications with a noticeable reduction of the rash and inflammation. During the fourth level of application the first areas of granulation tissue appeared and by the end of the eighth cycle, the therapy concluded with the lesion completely re-epitelized. Platelet gel showed to be effective and accomplished a complete re-epitelization of the lesion after eight cycles. At the same time, it allowed us to proceed with the myeloablative chemotherapy, which was the most appropriate therapeutic choice for the Hodgkin's disease but was supposed to enhance the risk of infective complications of the ulcer.
\end{abstract}

Keywords: Decubitus ulcer; Autologous PRP treatment; Hematologic patient

\section{Introduction}

Radiotherapy is an essential part of the multilevel treatment for Hodgkin's Lymphoma. However, this process is responsible for several adverse effects on different tissues. When combined with chemotherapy it makes normal tissues more sensible to radiations, and this may increase the risk of complications in relation to radiotherapy $[1,2]$.

The tissue damage may occur immediately or over a prolonged period of time $[2,3]$. Early damage is characterized by erythema and radionecrosis or delay in healing the wound. Late damage results in tissue necrosis. delayed wound healing, leading to trauma, ulcer or potential malignant growth in the irradiated tissue [3]. Several types of advanced dressings may be used alone or in combination with treatment for skin ulcers. Platelet-rich plasma (PRP) can be used for treating skin ulcers with different origin $[4,5]$.

\section{Case Report}

A 33 years old woman, diagnosised as stage 2A Hodgkin lymphoma nodular sclerosis histotype II grade according to WHO classification.Once diagnosed, in 2007, the patient had been treated with four cycles of chemotherapy (ABVD protocol)(ref) associated with radiotherapy at mediastinal, supraclavear and right axillary regions (30 Gy for each site) obtaining a complete remission. The patient suffered disease relapse and was treated with 4 cycles of salvage chemotherapy (IGEV schedule)(ref) In June 2010 .

There was a persistant residual disease documented by PET TC, between December 2010 and May 2011 and the patientwas submitted to a double autologous peripheral blood stem cell transplantation, with BEAM as conditioning regimen for the first procedure and MEL 200 for the second one. Currently the patient is well in complete remission of Hodgkin's disease. The patient suffered sternal pain and local rash at the end of radiotherapy. Sternal depression in the same region was noticed with several blister. These lesions evolved, into an ulcer involving cutaneous and subcutaneous layers.

Several cultural cell tests resulted positive for Corynebacterium, Staphylococcus Aureus and Enterobacteriacee. Thus, a targeted antibiotic therapy (Teicoplanin) was administered, associated with negative pressure therapy (VAC). Further cultural controls made until it confirms positive to Pseudomonas aeruginosa, Staphylococcus coagulase-negative, Staphylococcus aureus, Bacteroides and Corynebacterium.

The patient received a prolonged antibiotic therapy with different compounds (Ciprofloxacin, Linezolid, Daptomicine, Piperacillin+ Tazobactam) and the biopsy showed the presence of Hodgkin/ReedSternberg cells (CD-30+, CD-15-, CD-20-, EBV-) in the context of the ulcer. The patient was admitted in the Hematology department for the autologous transplantation, and the ulcer was of $6 \times 5 \mathrm{~cm}, 2 \mathrm{~cm}$ deep, of circular shape with uneven margins during the physical 
examination. There was a rash around the lesion and the wound was infected with abundant serous and fibrinous exudate in the center and squamous-crostous formations around the center.

The lesion was bleeding and was bed-smelling (Figure 1). We have decided to use applications of Platelet-rich plasma (PRP), with autologous plasma, throughout the patient's stay in the hospital and even for the outpatient treatment due to the bad results of the previous treatments. Neutropenia antibiotic therapy in association with i.v. Piperacillin/Tazobactam and Levofloxacin initially and i.v. Meropenem/Linezolid have been continued throughout chemotherapy treatment and after the onset of fever and pneumonitis.

The platelet gel has been applied once a week and the results were analyzed after 3 days with a view to evaluate the conditions of the medication. After the disinfection of the region with Sodio hypochlorite $0.05 \%$ cutaneous solution (Amukine Med 0.05\%, Amuchina S.p.A., Genova-Italy) and cutaneous solution based on $10 \%$ povidone iodine (Betadine ${ }^{\bullet} 10 \%$, Meda Pharma S.p.A., Milano-Italy) and the detersion with physiologic solution, we applied the gel on the ulcer and covered the lesion with hyaluronic acid-based lints, silver sulphadiazin $1 \%$ and sterile lints and plasters (Figure 2). The situation improved a lot even after the first two applications with a noticeable reduction of the rash and inflammation (Figure 3 ). At the end of the fourth application the granulation tissue appeared and at the end of eight cycles of therapy the lesion was completely re-epitelized (Figure $4)$.

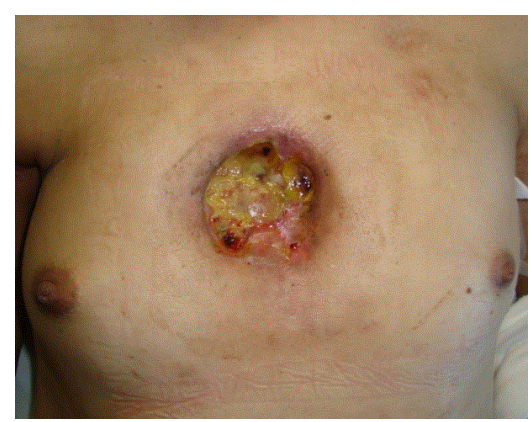

Figure 1: Lesion with bleeding.

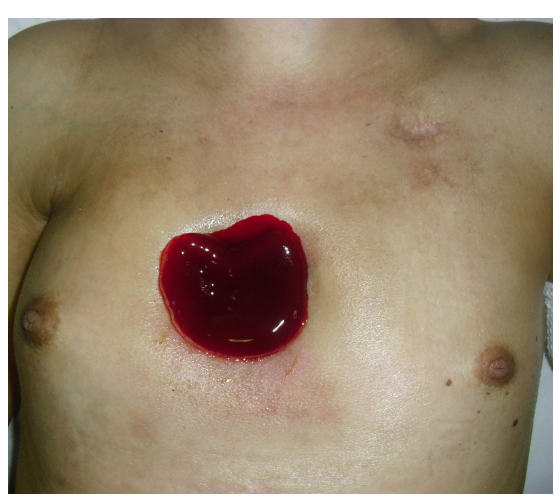

Figure 2: Lesion covered with hyaluronic acid-based lints.

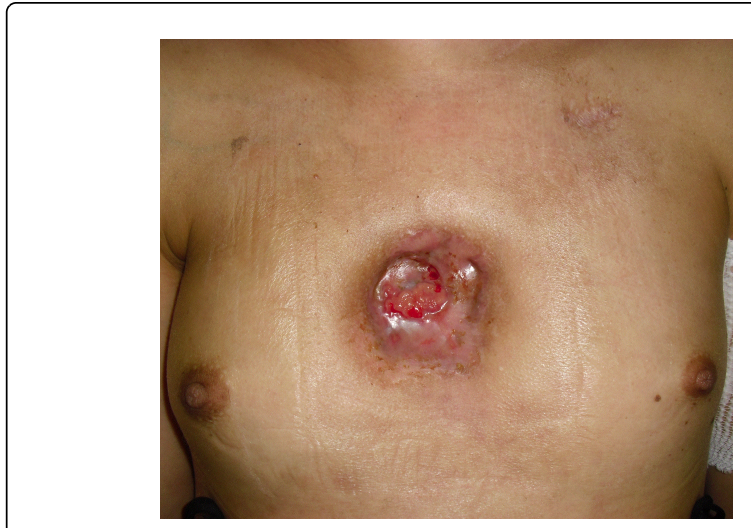

Figure 3: Reduction of the rash and inflammation.

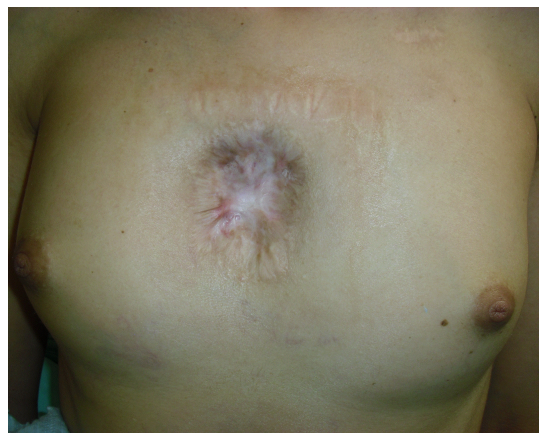

Figure 4: The ulcer after eight cycles of therapy the lesion was completely re-epitelized.

\section{Production of Hemocomponent}

The platelet gel was obtained with an autologous automatic procedure with multicomponent collection using Haemonetics $\mathrm{MCS}+{ }^{\circ}$ (Haemonetics Corp., Braintree, MA, USA) cell separator and a disposable (code 971E) for peripheral blood stem cell collection using a modified protocol. After about one hour, $60 \mathrm{ml}$ of leucoplatelet concentrate and almost $200 \mathrm{ml}$ of plasma were produced. The platelet count was significantly high, with more than $4800 \times 10^{3} / \mu \mathrm{l}$, WBC count was 10 times higher than the baseline values of peripheral blood. Cryoprecipitate was obtained from plasma thawed to $4^{\circ} \mathrm{C}$ overnight. This fraction was mixed with leucoplatelet concentrate to enrich the fibrinogen and other extracellular matrix proteins (fibronectine) and split into 8 small bags.

In this way, it is possible to get a topical emocomponent for several applications with one autologous procedure. The leucoplatelet GEL was made of autologous thrombin, platelet concentratecryoprecipitated enriched and gluconate calcium.

Despite very low (30\%) haematocrit presence, we could reach high cellular concentration in patient without G-CSF stimulation with this very cheap autologous procedure. The patient could tolerate the utologous procedure very well and ther was no side effects in this regard. 


\section{Discussion}

The interest in the use of blood components for different purposes besides transfusion therapy has been greatly expanded in recent years. The PRP is defined as a proportion of plasma containing a high concentration of platelets obtained from autologous blood [6]. PRP is used, since 1985 11, in several medical specialties [7-9].

PRP can be used in the treatment of skin ulcers with different origin in combination with other possible solutions: dressings with hyaluronic acid, modulators of proteases, collagen, and artificial tissues and, finally, stem cells of adipose tissue [4]. In all these wounds a positive response occurred in term of area and volume reduction [10].

The use of PRP is based on the recent knowledge about the platelet's role in healing processes. Platelets, in fact, are capable of processing, storing and releasing several factors that induce injured tissue's regeneration [5] and have both mitogenic and chemotactic properties [7]. In addition, the use of PRP gel for skin lesions is favored by its plasticity and moldability when applied at the affected site.

Growth factors that are present in the a-granules of platelets include: Platelet-Derived Growth Factor (PDGF-AA, BB, and AB isomers), Transforming Growth Factor- $\beta$ (TGF- $\beta$ ), Platelet Factor 4 (PF4), Interleukin-1 (IL-1), Platelet-Derived Angiogenesis factor (PDAF), Vascular Endothelial Growth Factor (VEGF), Epidermal Growth Factor (EGF), Platelet-Derived Endothelial Growth Factor (PDEGF), Epithelial Cell Growth Factor (ECGF), Insulin-like Growth Factor (IGF 1-2), Osteocalcin (Oc), Osteonectin (On), Fibrinogen (Ff), Vitronectin (Vn), Fibronectin (Fn), and Thrombospondin-1 (TSP-1) [11]. These growth factors aid in healing by attracting undifferentiated cells in the newly formed matrix, triggering cell division $[8,9]$. In addition, the findings that the release of growth factors from $\alpha$-granules of activated platelets occur many hours after the affixing of the PRP reduces its status as medicine for not too frequent applications. Our protocol providesone application a week at the affected area where ulcer can be seen followed by some gap found fruitful to control the wound.

Furthermore, PRP interacting with macrophages may suppress cytokine release and limit inflammation, improving the tissue healing and regeneration [12]. It promotes new capillary growth 9 and accelerates epithelialization [13] in chronic wounds.

Finally, platelets in PRP also play a role in host defense mechanism at the wound site by producing signaling proteins that attract macrophages [14]; PRP also may contain a small number of leukocytes [14] that synthesize interleukins as part of a non-specific immune response.

Generally, two types of products, commercial and laboratory made transfusional ("home-made") products are used for such applications. Commercial ones are obtained working on a huge number of plasma units and contain important concentrations of fibrinogen (about $80-120 \mathrm{mg} / \mathrm{ml}$ ). They are safe because of the sterilization and virusinactivation processes, and easy to use, yet quite expensive.

Home-made products (like the one we used in our study) are obtained from single units of allogenic or autologous plasma using cryoprecipitation technique.

Another factor in favor of PRP use is less expensive than engineered artificial tissues (dermal substitutes) [15]. Ultimately, the PRP is contraindicated with recurrent and persistent infections of ulcer, necrotic tissue with vasculitic origin, critical ischemia and with ulcers smaller than $2 \mathrm{~cm}^{2}$.

\section{Conclusion}

The peculiarity of our clinical case is that though the patient underwent treatment during 2009 and 2011, this lesion did not heal and it grew bigger in size than at the beginning of treatment. With the infectious wound, patient was at a very bad condition.

The use of the platelet gel prooved to be effective and accomplished a complete re-epitelization of the lesion after eight cycles. At the same time, it allowed us to proceed with the myeloablative chemotherapy, which was the most appropriate therapeutic choice for the Hodgkin's Disease but was supposed to enhance the risk of infective complications of the ulcer.

\section{Acknowledgments}

The authors hereby declare that they do not have any potential conflict of interests and did not receive funding for this work from any of the following organizations: National Institutes of Health (NIH); Welcome Trust; Howard Hughes Medical Institute (HHMI) and other(s). Each author participated sufficiently in the work to take public responsibility for the content.

Special thanks to Dr. Franco Bartolomei for his help in preparing this manuscript.

\section{References}

1. Caporaso NE, Goldin LR, Anderson WF, Landgren O (2009) Current insight on trends, causes, and mechanisms of Hodgkin's lymphoma. Cancer J 15: 117-123.

2. Stone HB, Coleman CN, Anscher MS, McBride WH (2003) Effects of radiation on normal tissue: consequences and mechanisms. Lancet Oncol 4: 529-536.

3. Loos B, Kopp J, Hohenberger W, Horch RE (2007) Post-malignancy irradiation ulcers with exposed alloplastic materials can be salvaged with topical negative pressure therapy (TNP). Eur J Surg Oncol 33: 920-925.

4. de Leon JM, Driver VR, Fylling CP, Carter MJ, Anderson C, et al. (2011) The clinical relevance of treating chronic wounds with an enhanced nearphysiological concentration of platelet-rich plasma gel. Adv Skin Wound Care 24: 357-368.

5. Everts PA, Brown Mahoney C, Hoffmann JJ, Schönberger JP, Box HA, et al. (2006) Platelet-rich plasma preparation using three devices: implications for platelet activation and platelet growth factor release. Growth Factors 24: 165-171.

6. Marx RE (2001) Platelet-rich plasma (PRP): what is PRP and what is not PRP? Implant Dent 10: 225-228.

7. Mehta S, Watson JT (2008) Platelet rich concentrate: basic science and current clinical applications. J Orthop Trauma 22: 432-438.

8. Fréchette JP, Martineau I, Gagnon G (2005) Platelet-rich plasmas: growth factor content and roles in wound healing. J Dent Res 84: 434-439.

9. Eppley BL, Pietrzak WS, Blanton M (2006) Platelet-rich plasma: a review of biology and applications in plastic surgery. Plast Reconstr Surg 118: 147e-159e.

10. Sell SA, Ericksen JJ, Reis TW, Droste LR, Bhuiyan MB, et al. (2011) A case report on the use of sustained release platelet-rich plasma for the treatment of chronic pressure ulcers. J Spinal Cord Med 34: 122-127.

11. Lacci KM, Dardik A (2010) Platelet-rich plasma: support for its use in wound healing. Yale J Biol Med 83: 1-9.

12. Mishra A, Woodall J Jr, Vieira A (2009) Treatment of tendon and muscle using platelet-rich plasma. Clin Sports Med 28: 113-125. 
Citation: Fioramonti P, Fino P, Ferrazza G, Capria V, Onesti MG (2015) Platelet-Rich Plasma (PRP) in a Post Radiotherapy Sternal Ulcer in a Patient with Hodgkin's Lymphoma. J Blood Disord Transfus 6: 299. doi:10.4172/2155-9864.1000299

Page 4 of 4

13. Knighton DR, Ciresi KF, Fiegel VD, Austin LL, Butler EL (1986) Classification and treatment of chronic nonhealing wounds. Successful treatment with autologous platelet-derived wound healing factors (PDWHF). Ann Surg 204: 322-330.
14. Gonshor A (2002) Technique for producing platelet-rich plasma and platelet concentrate: background and process. Int J Periodontics Restorative Dent 22: 547-557.

15. Parazzi V, Lazzari L, Rebulla P (2010) Platelet gel from cord blood: a novel tool for tissue engineering. Platelets 21: 549-554. 